\title{
IBS and Potential Luminosity Improvement for RHIC Operation Below Transition Energy
}

\author{
A. Fedotov
}

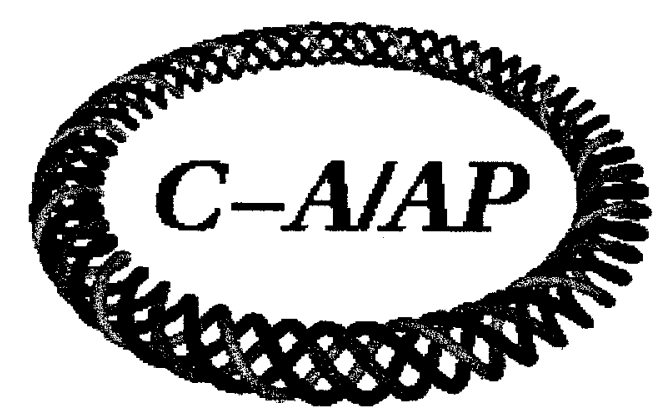

Collider-Accelerator Department Brookhaven National Laboratory

Upton, NY 11973

Notice: This document has been authorized by employees of Brookhaven Science Associates, LLC under Contract No. DE-AC02-98CH10886 with the U.S. Department of Energy. The United States Government retains a nonexclusive, paid-up, irrevocable, world-wide license to publish or reproduce the published form of this document, or allow others to do so, for United States Government purposes. 


\title{
IBS AND POTENITAL LUMINOSITY IMPROVEMENT FOR RHIC OPERATION BELOW TRANSITION ENERGY
}

\author{
Alexei Fedotov \\ $C-A D$, Brookhaven National Laboratory
}

\section{Introduction}

There is a strong interest in low-energy RHIC operations in the single-beam total energy range of 2.5-25 GeV/nucleon [1-3]. Collisions in this energy range, much of which is below nominal RHIC injection energy, will help to answer one of the key questions in the field of QCD about the existence and location of a critical point on the QCD phase diagram [4]. There have been several short test runs during 2006-2008 RHIC operations to evaluate RHIC operational challenges at these low energies [5]. Beam lifetimes observed during the test runs were limited by machine nonlinearities. This performance limit can be improved with sufficient machine tuning. The next luminosity limitation comes from transverse and longitudinal Intra-beam Scattering (IBS), and ultimately from the space-charge limit. Detailed discussion of limiting beam dynamics effects and possible luminosity improvement with electron cooling can be found in Refs. [6-8].

For low-energy RHIC operation, particle losses from the RF bucket are of particular concern since the longitudinal beam size is comparable to the existing RF bucket at low energies. However, operation below transition energy allows us to exploit an Intra-beam Scattering (IBS) feature that drives the transverse and longitudinal beam temperatures towards equilibrium by minimizing the longitudinal diffusion rate using a high RF voltage. Simulation studies were performed with the goal to understand whether one can use this feature of IBS to improve luminosity of RHIC collider at low-energies. This Note presents results of simulations which show that additional luminosity improvement for low-energy RHIC project may be possible with high RF voltage from a $56 \mathrm{MHz}$ superconducting RF cavity that is presently under development for RHIC.

\section{IBS below transition energy}

IBS is one of the major effects contributing to RHIC heavy ion luminosity degradation, driving bunch length and transverse beam emittance growth. IBS-driven bunch length growth causes beam losses from the RF bucket. For low-energy RHIC program longitudinal IBS becomes even more of a concern because the RF bucket acceptance is very small. For example, for lowest energy point from the proposed energy scan with $\gamma=2.7$, the $28 \mathrm{MHz}$ RF bucket acceptance with $300 \mathrm{kV}$ is only $0.08 \mathrm{eV}-\mathrm{s} /$ nucleon (maximum possible total voltage is $500 \mathrm{kV}$ which gives bucket acceptance of 0.1 $\mathrm{eV}-\mathrm{s} / \mathrm{n})$.

Longitudinal bunch emittance injected into AGS is about or less than $0.08 \mathrm{eV}-\mathrm{s} / \mathrm{n}$. However, it is increased significantly as a result of bunch merging and energy ramp. Recently, it was shown that emittance increase during merging can be controlled and kept to about $0.1 \mathrm{eV}-\mathrm{s} / \mathrm{n}$ at injection energy of AGS [9]. But emittance growth on the ramp still remains, resulting in emittance of about $0.2 \mathrm{eV}-\mathrm{s} / \mathrm{n}$ at extraction energies in a range of $\gamma=2-4$ and about $0.3 \mathrm{eV}-\mathrm{s} / \mathrm{n}$ for typical higher 
extraction energies. The work is presently underway to understand and minimize such longitudinal emittance growth on the ramp in AGS [9].

For low-energy RHIC program the goal is to have longitudinal emittance from AGS sufficiently small so that it fits into the RHIC RF bucket, thus minimizing intensity loss at injection in RHIC. As an example, Table 1 lists RF bucket acceptance for present $28 \mathrm{MHz}$ RF with maximum possible total gap voltage of $500 \mathrm{kV}$ for the lowest energy points of interest. Only harmonic numbers which are divisible by 9 (which satisfy both of present constraints of experiment trigger and geometry of RHIC experiments [10]) are shown.

\begin{tabular}{|l|l|l|}
\hline$\gamma$ & h & RF bucket acceptance, eV-s/nucleon \\
\hline 2.7 & 387 & 0.1 \\
\hline 3.2 & 378 & 0.14 \\
\hline 4.3 & 369 & 0.23 \\
\hline
\end{tabular}

Table 1. $28 \mathrm{MHz}$ RF (500kV total gap voltage) bucket acceptance.

Due to the longitudinal IBS, strong debunching will occur even with perfect longitudinal injection. In fact, getting longitudinal emittances from AGS significantly smaller than RF bucket acceptance in RHIC does not help much because the longitudinal IBS only becomes stronger resulting is strong debunching (unless electron cooling in RHIC is provided $[6,7]$ ). For example, Table 2 shows how longitudinal IBS rate would increase if one would inject bunches with smaller longitudinal momentum spread. Actually, at one point, an option of obtaining such small longitudinal emittances with pre-cooling at AGS injection was discussed.

However, below transition energy IBS drives the transverse and longitudinal beam temperatures towards equilibrium (in a smooth lattice approximation). This suggests that longitudinal heating can be stopped (at least initially) if the longitudinal beam temperature is larger than transverse, at the expense of the transverse heating, of course. If there is enough RF voltage, one can increase longitudinal beam temperature by shrinking the bunch length or perhaps inject the beam with larger longitudinal emittance (provided that RF bucket acceptance is sufficiently large). By doing so one can then redistribute IBS between the longitudinal and transverse degrees of freedom and minimize losses from RF bucket due to the longitudinal IBS. Experimental study of such redistribution effect due to IBS below transition could be found, for example, in Ref. [11].

Unfortunately, to produce sufficiently large momentum spread with $28 \mathrm{MHz} \mathrm{RF}$ would require larger RF voltage than we presently have (this is in the assumption that transverse beam emittances will be close to typical values at RHIC injection energy). The maximum possible total RF gap voltage on two cavities is now $500 \mathrm{kV}$ (the use of $450 \mathrm{kV}$ with beam operation was demonstrated). Table 3 shows that having $1 \mathrm{MV}$ on $28 \mathrm{MHz}$ RF would allow us to accommodate large incoming longitudinal emittances (up to $0.145 \mathrm{eV}$-s/nucleon for the lowest point of interest with $\gamma=2.7$ ) and provide sufficiently large longitudinal beam temperature. Alternatively, for small longitudinal emittance of $0.1 \mathrm{eV}-\mathrm{s} /$ nucleon, RF voltage of $1 \mathrm{MV}$ would allow us to shrink the bunch length and increase rms momentum spread to 0.00058 which is close to the values needed to stop longitudinal IBS heating and thus intensity loss from the RF bucket due to IBS (see Table 3). 


\begin{tabular}{|l|l|l|l|}
\hline $\mathrm{S}_{95 \%}, \mathrm{eV}-\mathrm{s} /$ nucleon & $\sigma_{\mathrm{p}}$ & transverse IBS $\tau_{\mathrm{x}}{ }^{-1}, \mathrm{sec}^{-1}$ & longitudinal IBS $\tau_{\mathrm{z}}{ }^{-1}, \mathrm{sec}^{-1}$ \\
\hline 0.09 & 0.00045 & 0.007 & 0.006 \\
\hline 0.07 & 0.0004 & 0.0065 & 0.014 \\
\hline 0.04 & 0.0003 & 0.004 & 0.06 \\
\hline
\end{tabular}

Table 2. IBS rates $\left(\tau_{\mathrm{x}}{ }^{-1}=\mathrm{d} \varepsilon_{\mathrm{x}} / \varepsilon_{\mathrm{x}} \mathrm{dt}, \tau_{\mathrm{z}}{ }^{-1}=\mathrm{d} \sigma_{\mathrm{p}}{ }^{2} / \sigma_{\mathrm{p}}{ }^{2} \mathrm{dt}\right)$ for different longitudinal momentum spread (beam temperature) for $28 \mathrm{MHz} \mathrm{RF}$ with $500 \mathrm{kV}$ total gap voltage, RF bucket acceptance $0.1 \mathrm{eV}$-s/nucleon, $\gamma=2.7$, bunch intensity $N=1 \times 10^{9}$, transverse beam emittance of $\varepsilon=15 \mu \mathrm{m}(95 \%$, normalized).

In this Note, we use convention of $95 \%$ longitudinal emittance defined as:

$$
S_{95 \%}=6 \pi \frac{m c^{2}}{c}(\beta \gamma) \sigma_{p} \sigma_{z}
$$

where $m$ is the rest mass per nucleon, $\sigma_{z}$ is rms bunch length and $\sigma_{p}$ is rms momentum spread.

\begin{tabular}{|l|l|l|l|}
\hline $\mathrm{S}_{95 \%}, \mathrm{eV}-\mathrm{s} /$ nucleon & $\sigma_{\mathrm{p}}$ & transverse IBS $\tau_{\mathrm{x}}^{-1}, \mathrm{sec}^{-1}$ & longitudinal IBS $\tau_{\mathrm{z}}^{-1}, \mathrm{sec}^{-1}$ \\
\hline 0.11 & 0.0006 & 0.001 & 0 \\
\hline 0.13 & 0.00065 & 0.009 & -0.0015 \\
\hline
\end{tabular}

Table 3. IBS rates $\left(\tau_{\mathrm{x}}{ }^{-1}=\mathrm{d} \varepsilon_{\mathrm{x}} / \varepsilon_{\mathrm{x}} \mathrm{dt}, \tau_{\mathrm{z}}{ }^{-1}=\mathrm{d} \sigma_{\mathrm{p}}{ }^{2} / \sigma_{\mathrm{p}}{ }^{2} \mathrm{dt}\right)$ for $28 \mathrm{MHz}$ RF with 1MV total gap voltage, bucket acceptance $0.145 \mathrm{eV}$-s/nucleon, $\gamma=2.7$, bunch intensity $\mathrm{N}=1 \times 10^{9}$ and transverse beam emittance $\varepsilon=15 \mu \mathrm{m}(95 \%$, normalized $)$.

An idea of employing IBS redistribution below transition energy to minimize longitudinal IBS is rather simple. But for RHIC application we are interested in maximizing the luminosity. This means that not just the bunch length growth and intensity loss due to debunching but also the transverse emittance growth is important as well. Therefore, it is essential to understand whether stopping longitudinal IBS at the expense of the transverse emittance growth actually buy us anything in terms of luminosity improvement or not. To see what luminosity gain one can obtain with such IBS redistribution, beam dynamics simulations were performed with the BETACOOL code [12]. Once again, we remind that providing electron cooling at these low energies in RHIC would not have this problem of a trade between different degrees of freedom since both the transverse and longitudinal IBS would be counteracted $[6,7]$.

Effect of IBS redistribution and its impact on luminosity improvement was first studied with $28 \mathrm{MHz}$ and then with the proposed $56 \mathrm{MHz}$ RF. Since $28 \mathrm{MHz}$ RF maximum available voltage is limited to $500 \mathrm{kV}$ while simulations showed that we would need up to $1 \mathrm{MV}$ (for transverse emittances of $15 \mu \mathrm{m}$ ), we omit that studies from present Note and summarise in the next section our results for the $56 \mathrm{MHz} \mathrm{RF}$ only.

\section{Operation with 56MHz RF for RHIC low-energies}

An upgrade of RHIC storage RF system with $56 \mathrm{MHz}$ superconducting cavity is presently underway [13]. With $2.5 \mathrm{MV}$ voltage this cavity will provide large bucket acceptance and will significantly improve RHIC performance at top energy both for heavy ions and protons [14].

For low-energy operation (below transition energy) one can find harmonic numbers which should allow us to use this SRF cavity for fixed RF frequency. Table 4 shows several energies available for 
fixed RF frequency of $56.2989 \mathrm{MHz}$ by choosing appropriate RF harmonic. Only harmonics which are divisible by 9 , which satisfy present timing and experiment constraints, are shown. If these constraints [10] are removed it may allow us to use other harmonic numbers and energies correspondingly.

\begin{tabular}{|l|l|l|}
\hline$\gamma$ & h & RF bucket acceptance, eV-s/nucleon \\
\hline 6.4 & 729 & 0.34 \\
\hline 4.6 & 738 & 0.2 \\
\hline 3.8 & 747 & 0.14 \\
\hline 3.3 & 756 & 0.12 \\
\hline 3.0 & 765 & 0.1 \\
\hline 2.7 & 774 & 0.08 \\
\hline
\end{tabular}

Table 4. Harmonic numbers and energies for low energies with fixed RF frequency of $56.2989 \mathrm{MHz}$ (with 2.5MV RF voltage)

One can see (Table 4) that use of 56MHz RF with 2.5MV does not give us an improvement in terms of bucket acceptance compared to $28 \mathrm{MHz}$ RF with $0.5 \mathrm{MV}$ (see Table 1). This is because the RF bucket acceptance $A_{s}$ scales with RF harmonic and voltage as

$$
A_{s}[e V-s / \text { nucleon }]=8 \frac{R}{h c} \sqrt{\frac{2 Z e V_{r f} E_{s}}{\pi h \eta A}} \text {, }
$$

where $R$ is the machine radius, $h$ is the RF harmonic number, $\eta$ is the slippage factor, $V_{r f}$ is the total RF gap voltage per turn, $Z$ is the ion charge number, $A$ is atomic mass and $E_{s}$ is the energy of synchronous particles in units eV/nucleon. The control of the longitudinal emittance in AGS is thus important for injection into RHIC RF at low-energies, since we do not want to loose bunch intensity and peak luminosity right away as a result of injection, if incoming longitudinal emittance is larger than the bucket acceptance.

However, the bunch length with $56 \mathrm{MHz}$ RF will be shorter than with $28 \mathrm{MHz}$ RF which will provide us with larger momentum spread for the same longitudinal emittance. This is beneficial both for IBS redistribution (as discussed in previous section) as well as for the useful luminosity within the detector vertex.

\begin{tabular}{|l|l|l|}
\hline$\gamma$ & $\mathrm{S}_{95 \%}, \mathrm{eV}$-s/nucleon & Full bunch length in AGS, nsec \\
\hline 2.7 & 0.1 & 32 \\
\hline & 0.2 & 45 \\
\hline 3.2 & 0.1 & 27 \\
\hline & 0.2 & 39 \\
\hline & 0.1 & 21 \\
\hline 6.4 & 0.2 & 29 \\
\hline & 0.1 & 13 \\
\hline
\end{tabular}

Table 5. Expected bunch length in AGS at RF harmonic $\mathrm{h}=12$ with $260 \mathrm{kV}$ gap voltage.

There are, of course, RF issues related to injection into RHIC $56 \mathrm{MHz}$ RF at these low energies which will need to be addressed. For example, even for relatively high energy point from the energy scan proposed with $\gamma=6.4$ and incoming beam with the longitudinal emittance of $0.2 \mathrm{eV}-\mathrm{s} / \mathrm{n}$, the 
bunch length from AGS RF is about 19 nsec. The length of incoming bunch from AGS is even longer for the lower energy points. This is too long to inject directly into $56 \mathrm{MHz}$ RF in RHIC which has only 18 nsec bucket length. The expected bunch length at different energy points are summarized in Table 5 for convenience.

Two approaches were discussed to resolve this issue. The first one is so-called "quad pumping" when coherent longitudinal oscillations are excited in AGS and the bunch is extracted to RHIC when the bunch length is at its minimum. However, this approach would be limited to 1 bunch extraction mode. Another approach, which looks simpler at this stage, is to inject first into $28 \mathrm{MHz}$ $\mathrm{RF}$ (bucket length $36 \mathrm{nsec}$ ) and then adiabatically bring on $56 \mathrm{MHz}$ RF voltage which will shorten the bunch length. In this latter case, only selected $56 \mathrm{MHz}$ RF harmonics could be used.

Note, that even for injection in $28 \mathrm{MHz}$ RF with bucket length of $36 \mathrm{nsec}$, one would like to have longitudinal emittance below $0.14 \mathrm{eV}$-s/nucleon for $\gamma<3.5$ (see Table 5). On the other hand, it may be beneficial to have longitudinal emittances of $0.2 \mathrm{eV}$-s/nucleon or larger for $\gamma>4.3$, in order to ensure small longitudinal IBS growth during accumulation process. To inject 56 bunches in both RHIC rings before bringing on adiabatically $56 \mathrm{MHz}$ RF will take about $100 \mathrm{sec}$. It is thus desired that longitudinal IBS growth times are significantly less than this time to reduce debunching during accumulation process.

Table 6 shows expected IBS growth rates for different longitudinal emittance of incoming bunch at several energies. Calculation of IBS rates were done with an assumption that the transverse emittance of incoming bunch is $15 \mu \mathrm{m}$ (95\%, normalized) at any energy. If transverse emittance is larger at lower energy points, then the ratio between the transverse and longitudinal beam temperatures will be different and IBS rates will need to be recalculated. Note, that for the case of $\gamma=6.4$ an attempt to increase longitudinal emittance in order to reduce longitudinal IBS resulted in beam temperatures close to equilibrium. As a result, both longitudinal and transverse IBS growth rates became very small. Such operational condition would be ideal, of course. Unfortunately, significant debunching will occur in this case since longitudinal emittance of $0.4 \mathrm{eV}-\mathrm{s} / \mathrm{n}$ is already close to RF bucket acceptance at this energy. Nevertheless, having sufficiently large longitudinal emittance may be beneficial to minimize longitudinal IBS during accumulation process.

\begin{tabular}{|l|l|l|l|l|}
\hline$\gamma$ & $\mathrm{h}$ & $\mathrm{S}_{95 \%}, \mathrm{eV}-\mathrm{s} / \mathrm{n}$ & $\tau_{\mathrm{x}}{ }^{-1}, \mathrm{sec}^{-1}$ & $\tau_{\mathrm{z}}{ }^{-1}, \mathrm{sec}^{-1}$ \\
\hline 2.7 & 387 & 0.1 & 0.007 & 0.004 \\
\hline 3.2 & 378 & 0.1 & 0.004 & 0.006 \\
\hline & & 0.14 & 0.0044 & 0.002 \\
\hline 4.3 & 369 & 0.1 & 0.0015 & 0.013 \\
\hline & & 0.14 & 0.0018 & 0.005 \\
\hline & & 0.2 & 0.002 & 0.0016 \\
\hline 6.4 & 363 & 0.1 & 0.0002 & 0.016 \\
\hline & & 0.2 & 0.0006 & 0.003 \\
\hline & & 0.4 & 0.0007 & 0.0006 \\
\hline
\end{tabular}

Table 6. Longitudinal $\tau_{\mathrm{z}}{ }^{-1}$ and transverse $\tau_{\mathrm{x}}{ }^{-1}$ IBS rates $\left(\tau_{\mathrm{x}}{ }^{-1}=\mathrm{d} \varepsilon_{\mathrm{x}} / \varepsilon_{\mathrm{x}} \mathrm{dt}, \tau_{\mathrm{z}}{ }^{-1}=\mathrm{d} \sigma_{\mathrm{p}}{ }^{2} / \sigma_{\mathrm{p}}{ }^{2} \mathrm{dt}\right)$ for different longitudinal emittance (beam temperature) for $28 \mathrm{MHz}$ RF with $500 \mathrm{kV}$ total gap voltage. Bunch intensity $\mathrm{N}=1 \times 10^{9}$, transverse beam emittance $\varepsilon=15 \mu \mathrm{m}(95 \%$, normalized). 


\section{Luminosity performance}

In this section we show results of simulation and discuss potential luminosity improvement for lowenergy RHIC operation. Figures 1-3 show comparison between independent operation with $28 \mathrm{MHz}$ and $56 \mathrm{MHz}$ RF for the lowest energy point of interest at $\gamma=2.7$. Simulations were done for the initial parameters of ion bunch given in Table 7. Longitudinal emittance of $\mathrm{S}_{95 \%}=0.1 \mathrm{eV}$-s/nucleon assumes that emittance growth on the ramp in AGS can be minimized [9]. The bunch intensity was kept at $\mathrm{N}=0.5 \times 10^{9}$ in order not to exceed space-charge limit in a collider mode $[7,8]$.

\begin{tabular}{|l|l|}
\hline$\gamma$ & 2.7 \\
\hline $\mathrm{N}, \times 10^{9}$ & 0.5 \\
\hline transverse emittance $\varepsilon(95 \%), \mu \mathrm{m}$ & 15 \\
\hline Longitudinal emittance $\mathrm{S}_{95 \%,}$ eV-s/nucleon & 0.1 \\
\hline
\end{tabular}

Table 7. Parameters of $\mathrm{Au}$ ion bunch used for simulations in Figs 1-3.

Figure 1 shows that in the case of $28 \mathrm{MHz}$ RF significant debunching occurs as a result of the longitudinal IBS, while for the case of $56 \mathrm{MHz}$ RF longitudinal IBS is initially stopped since longitudinal beam temperature is larger than transverse (this relation between longitudinal and transverse temperatures is changing with time due transverse emittance growth). Initial loss observed in Fig. 1 is due to the fact that RF bucket acceptance of $0.08 \mathrm{eV}-\mathrm{s} /$ nucleon for $56 \mathrm{MHz} \mathrm{RF}$ is slightly small. Note, that for simulations presented here we used linear RF bucket approximation in BETACOOL code. Implementation of non-linear RF in the code is presently under development. Simulations will be repeated in the future with the non-linear RF, which should provide more realistic beam loss from the RF bucket.

Figure 2 shows that there is practically no improvement in total average per store luminosity since in the case of $56 \mathrm{MHz}$ RF transverse emittance is growing much faster, as can be seen in Fig. 3. Despite the fact that there is no direct gain in total luminosity in Fig. 2, one can still expect to have up to a factor of two improvement in useful vertex luminosity because for the case of $56 \mathrm{MHz} \mathrm{RF}$ the bunch length is twice smaller than for the case of $28 \mathrm{MHz}$ RF. Note that this gain in luminosity will be limited to short store times due to beam loss on transverse acceptance, if transverse emittance is quickly growing. Simulations presented in this section do not include beam loss on the transverse acceptance with the purpose of transparent discussion of IBS redistribution. For actual expected luminosity gain, an optimum scenario between transverse and longitudinal IBS could be established for specific initial beam parameters, and simulations should be repeated including beam loss on the transverse acceptance. 


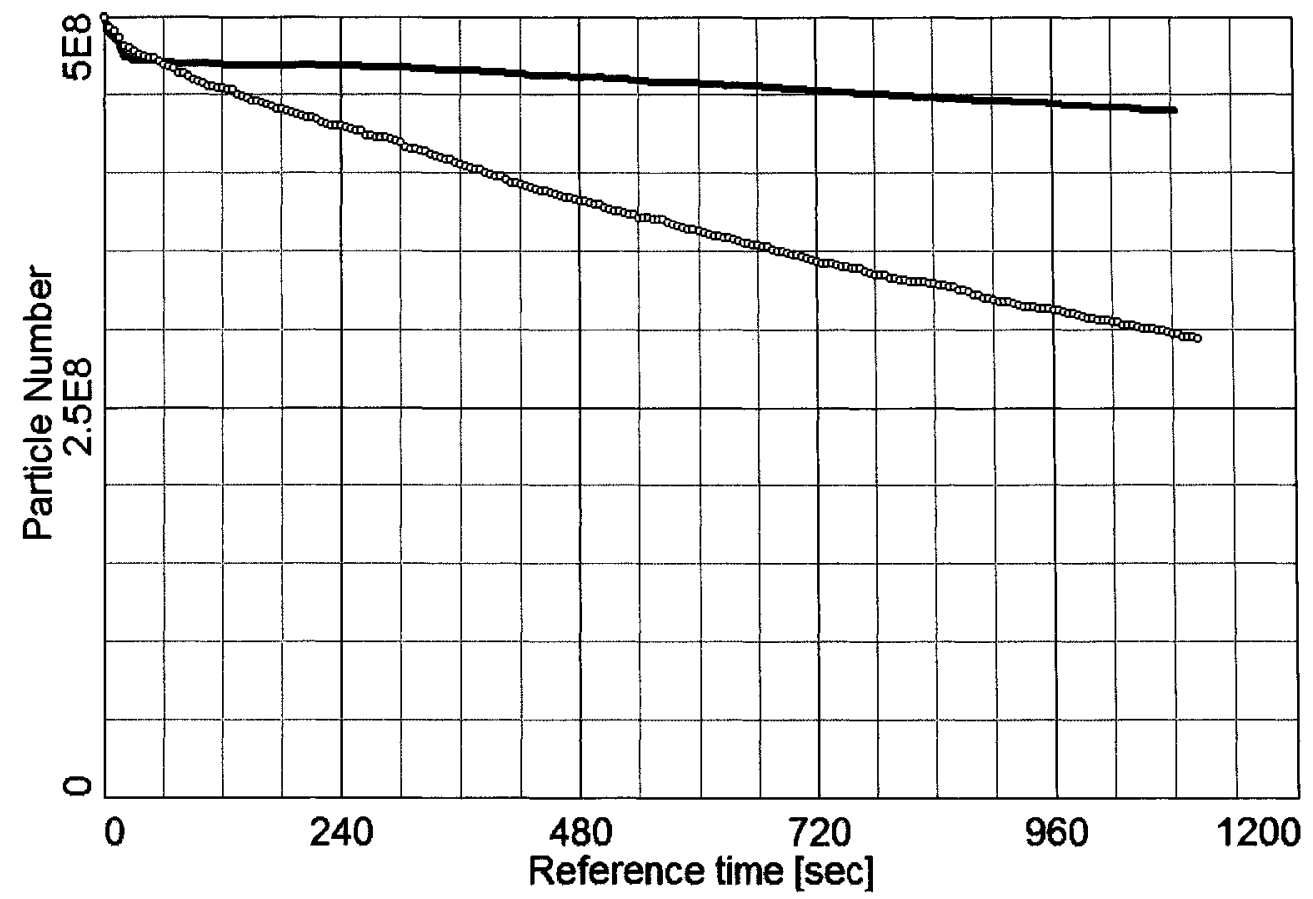

Figure 1. Bunch intensity loss at $\gamma=2.7$, for initial beam parameters in Table 7:1) upper curve - for $56 \mathrm{MHz}$ RF (2.5MV); 2) lower curve - for $28 \mathrm{MHz} \mathrm{RF}(0.5 \mathrm{MV})$.



Figure 2. Full luminosity for 56 bunches with $\beta^{*}=10 \mathrm{~m}$ and initial beam parameters listed in Table 7: 1) blue curve $-56 \mathrm{MHz}$ RF (2.5MV) 2) black curve $-28 \mathrm{MHz} \mathrm{RF}(0.5 \mathrm{MV})$. 




Figure 3. RMS un-normalized transverse emittances at $\gamma=2.7$ for initial beam parameters in Table 7: 1) upper blue/red curves $-56 \mathrm{MHz} \mathrm{RF}$; 2) lower black curve $-28 \mathrm{MHz} \mathrm{RF}$.

Figures 4-7 show comparison between independent operation with $28 \mathrm{MHz}$ and $56 \mathrm{MHz} \mathrm{RF}$ for higher energy point at $\gamma=6.3$. At this higher energy, we are not yet space-charge limited which allows us to use higher bunch intensities. Simulations were done for beam parameters in Table 8 . In this case, RF bucket acceptance is sufficiently large and we practically do not see debunching in Fig. 4 with $56 \mathrm{MHz}$ RF since longitudinal IBS is suppressed (initial longitudinal beam temperature is larger than transverse). Although small, an advantage in luminosity performance due to IBS redistribution can be seen directly in Fig. 5. Figure 7 shows that one can actually gain about a factor of two in vertex luminosity since for the case of $56 \mathrm{MHz}$ RF the bunch length is twice smaller than for the case of $28 \mathrm{MHz} \mathrm{RF}$.

\begin{tabular}{|l|l|}
\hline$\gamma$ & 6.3 \\
\hline $\mathrm{N}, \times 10^{9}$ & 1 \\
\hline transverse normalized emittance $\varepsilon(95 \%), \mu \mathrm{m}$ & 15 \\
\hline longitudinal emittance $\mathrm{S}_{95 \%}, \mathrm{eV}-\mathrm{s} /$ nucleon & 0.2 \\
\hline
\end{tabular}

Table 8. Parameters of $\mathrm{Au}$ ion bunch used in simulations in Figs. 4-7. 


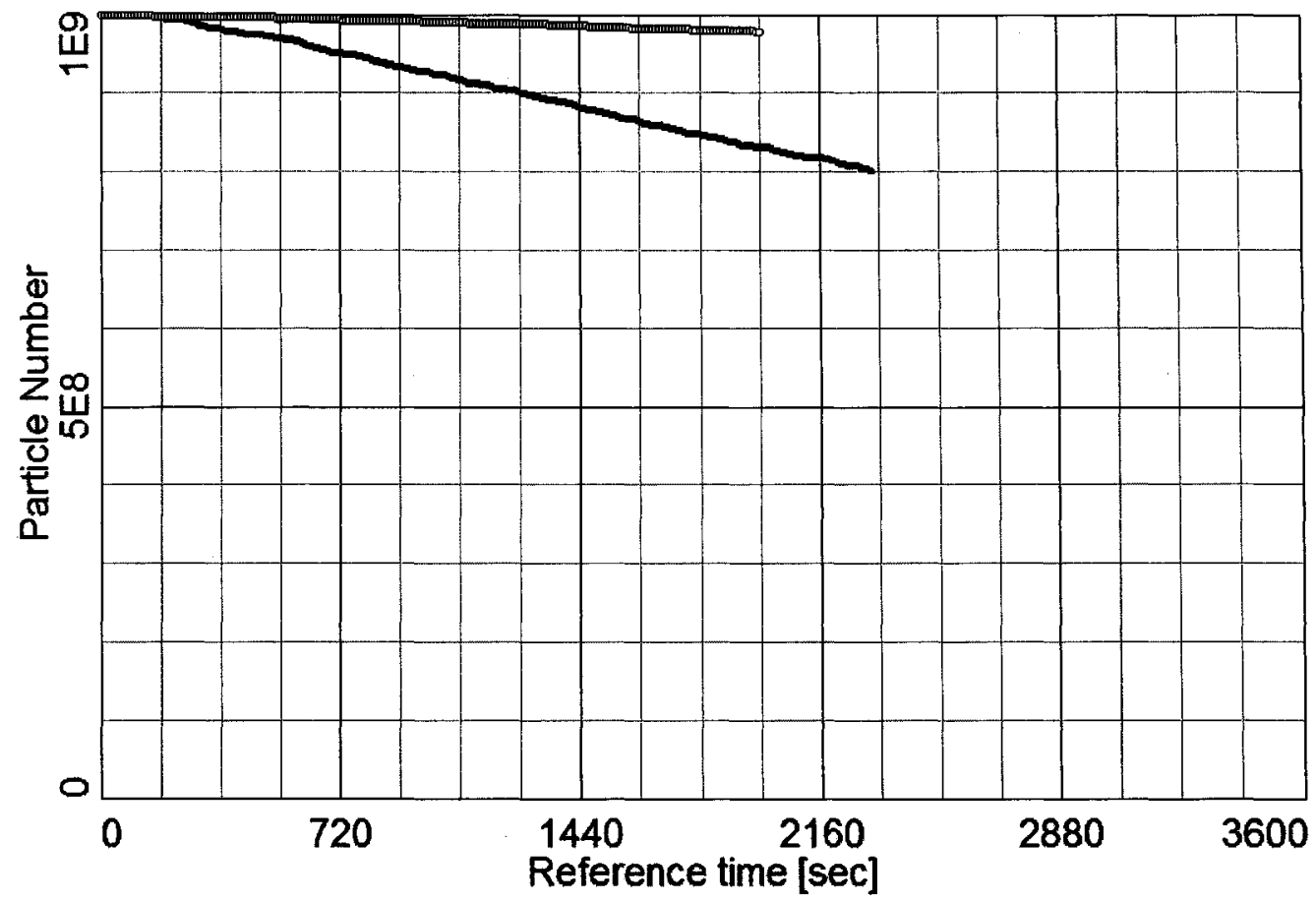

Figure 4. Bunch intensity loss at $\gamma=6.3$, for beam parameters in Table 8: 1) upper curve - for $56 \mathrm{MHz}$ $\mathrm{RF}(2.5 \mathrm{MV})$; 2) lower curve - for $28 \mathrm{MHz} \mathrm{RF}(0.5 \mathrm{MV})$.



Figure 5. Full luminosity for 56 bunches with $\beta^{*}=10 \mathrm{~m}$ and beam parameters in Table 8: 1) blue upper curve $-56 \mathrm{MHz}$ RF $(2.5 \mathrm{MV}) ; 2$ ) black lower curve $-28 \mathrm{MHz} \mathrm{RF}(0.5 \mathrm{MV})$. 


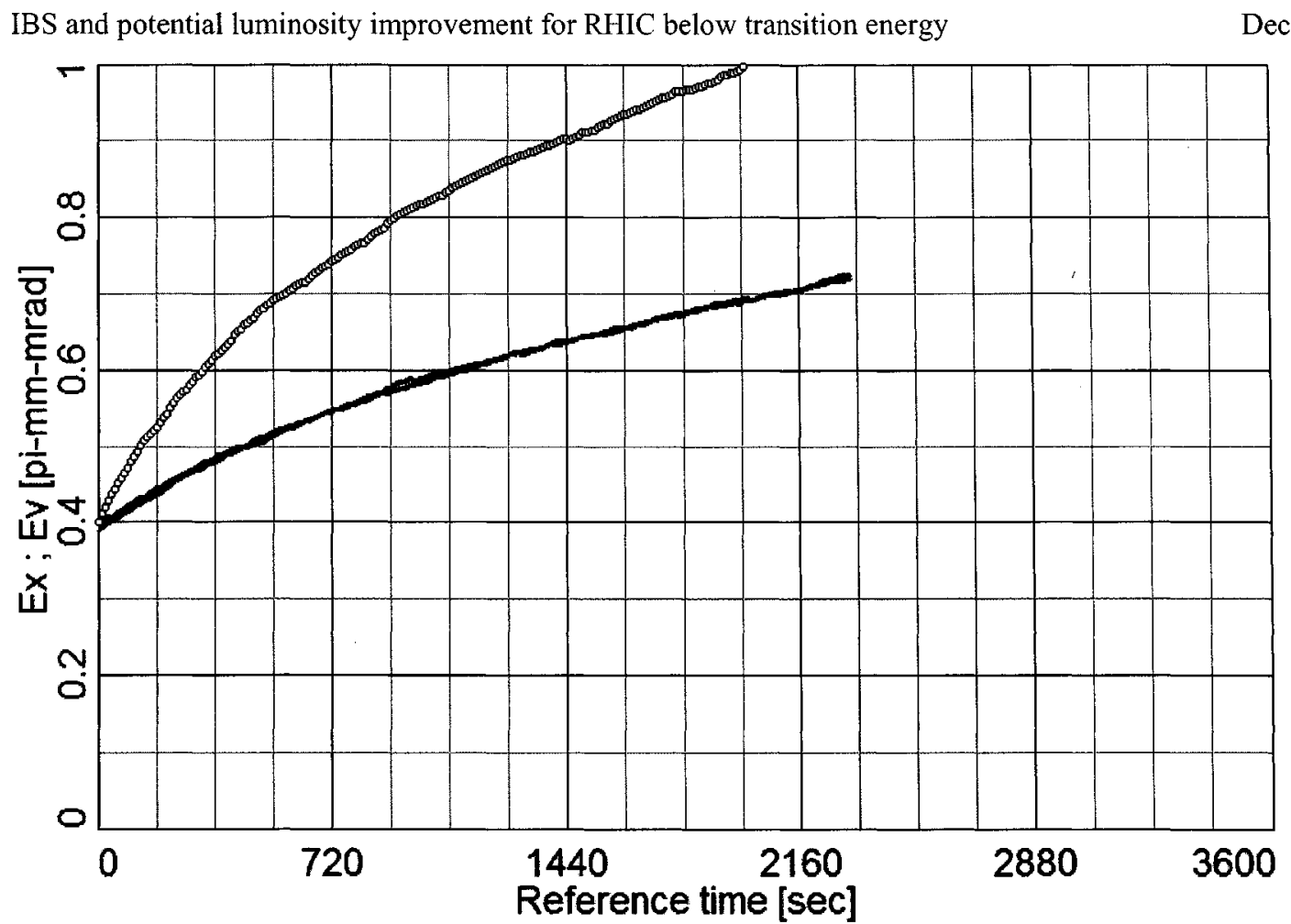

Figure 6. RMS un-normalized transverse emittances at $\gamma=6.3$ for beam parameters in Table 8: 1) upper black curve $-56 \mathrm{MHz}$ RF; 2) lower blue/red curve $-28 \mathrm{MHz}$ RF.

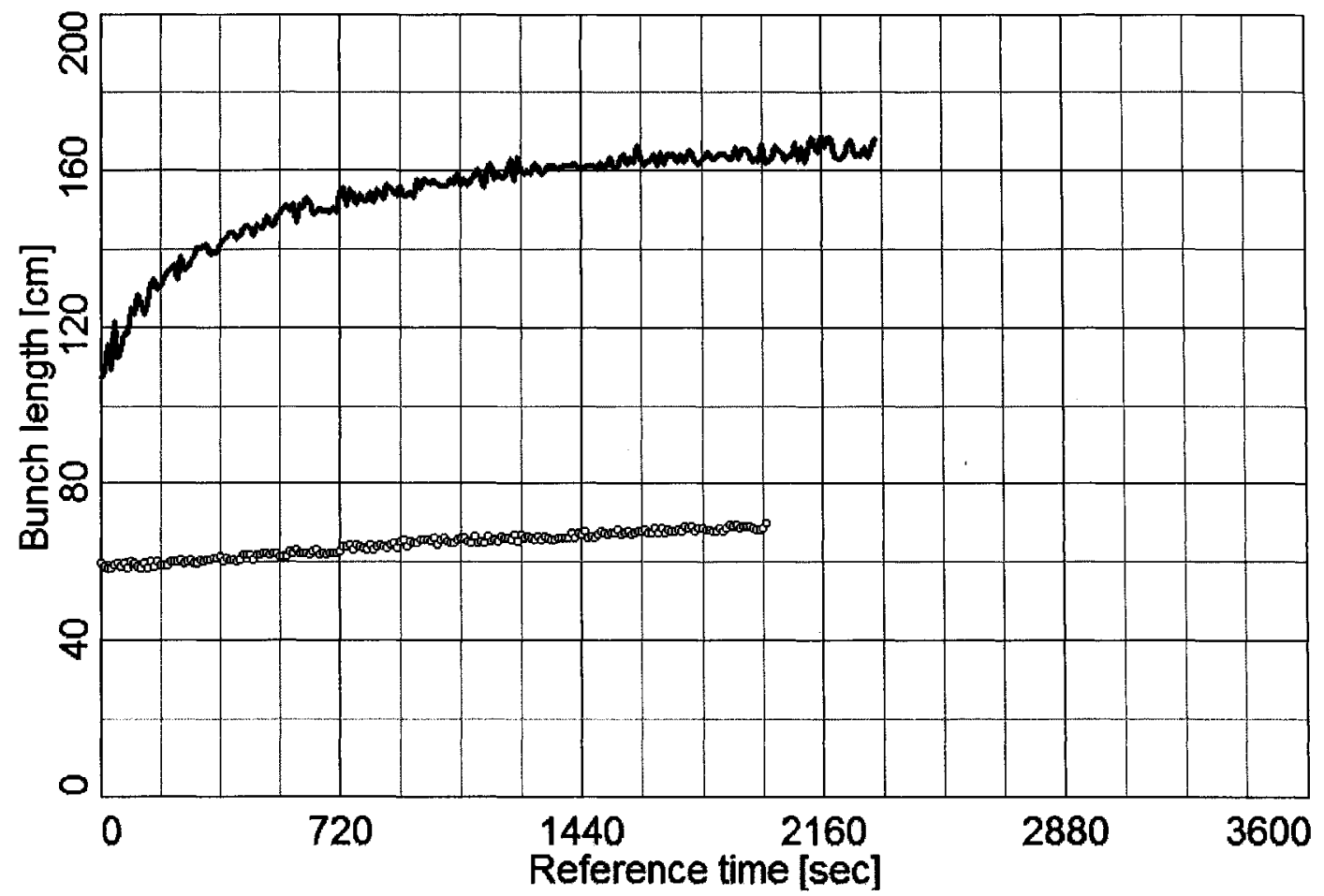

Figure 7. RMS bunch length at $\gamma=6.3$ for beam parameters in Table 8: 1) upper curve $-28 \mathrm{MHz} \mathrm{RF}$ (0.5MV gap voltage); 2 ) lower curve $-56 \mathrm{MHz}$ RF (2.5MV). 


\section{Summary}

An idea to use IBS redistribution below transition energy in order to stop bunch length growth and thus bunch intensity and luminosity loss due to the longitudinal IBS was explored. It was found that proposed $56 \mathrm{MHz}$ RF upgrade may provide RF voltage needed to minimize longitudinal growth rate due to IBS. A working scenario could be to inject first into $28 \mathrm{MHz}$ RF (bucket length $36 \mathrm{nsec}$ ) and then adiabatically bring on $56 \mathrm{MHz}$ RF voltage which will shorten the bunch length. Overall, about factor of two of improvement in useful vertex luminosity for RHIC operation below transition energy might be possible but store length for each specific energy will be limited by an amount of tolerable beam losses, including on the transverse acceptance. More realistic simulations are planned to quantify expected luminosity gains.

\section{Acknowledgement}

We would like to thank I. Ben-Zvi, M. Blaskiewicz, M. Brennan, D. Kayran, V. Litvinenko, E. Pozdeyev and T. Satogata for many useful discussions on this subject. In simulations, we used BETACOOL code developed at JINR, Dubna, Russia.

\section{References}

[1] Proc. of Workshop "Can we discover QCD critical point at RHIC?" (BNL, March 2006) RIKEN BNL Research Center Report No. BNL-75692-2006; http://www..bnl.gov/riken/QCDRhic.

[2] A. Cho, Science, V. 312, April 12, 2006, p 190.

[3] G. Stephans, J. Phys. G: Nucl. Part. Phys. 32 (2006).

[4] M. Stephanov, K. Rajagopal, and E. Shuryak, Phys. Rev. Letters 81, p. 4816 (1998).

[5] T. Satogata et al., "RHIC challenges for low energy operation", Proceed. of PAC07 (Albuquerque, NM, 2007), p. 1877; T. Satogata et al., 2008 RHIC retreat, March 31, 2008.

[6] A. Fedotov, I. Ben-Zvi, X. Chang, D. Kayran, T. Satogata, Proc. of COOL07 (Bad Kreuznach, Germany, 2007), p. 243.

[7] A. Fedotov, I. Ben-Zvi, X. Chang, D. Kayran, V. Litvinenko, E. Pozdeyev, T. Satogata, BNL Collider-Accelerator AP Note: C-A/AP/307 (April 2008).

[8] A. Fedotov, I. Ben-Zvi, X. Chang, D. Kayran, V. Litvinenko, E. Pozdeyev, T. Satogata, Proc. of HB2008 workshop (Nashville, TN 2008).

[9] E. Pozdeyev, RHIC retreat (March 31, 2008).

[10] T. Satogata, BNL C-AD Tech Note: C-A/AP/309 (May 2008).

[11] M. Hu and S. Nagaitsev, Proc. of PAC05 (Knoxville, TN, 2005), p. 1560.

[12] BETACOOL code, http://lepta.jinr.ru; A. Sidorin et al., NIM A 558, p. 325 (2006).

[13] http://www.bnl.gov/cad/ecooling.

[14] A. Fedotov, BNL C-AD Tech Note: C-A/AP/332 (October 2008). 